\title{
Characterization and video chain development of the CMOS detector applied in the multi-angle spectro- polarimeter SPEXone
}

Jochen Campo, Paul Tol, Joris van der Vlugt, Martijn Smit, Jelle Talsma, et al.

Jochen Campo, Paul Tol, Joris van der Vlugt, Martijn Smit, Jelle Talsma, Jens Johansen, Alexander Eigenraam, Richard van Hees, Otto Hasekamp, Marc Oort, Aaldert van Amerongen, Jeroen Rietjens, "Characterization and video chain development of the CMOS detector applied in the multi-angle spectropolarimeter SPEXone," Proc. SPIE 11852, International Conference on Space Optics — ICSO 2020, 1185213 (11 June 2021); doi: 10.1117/12.2599224

SPIE Event: International Conference on Space Optics - ICSO 2021, 2021, Online Only 


\section{International Conference on Space Optics-ICSO 2020}

Virtual Conference

30 March-2 April 2021

Edited by Bruno Cugny, Zoran Sodnik, and Nikos Karafolas
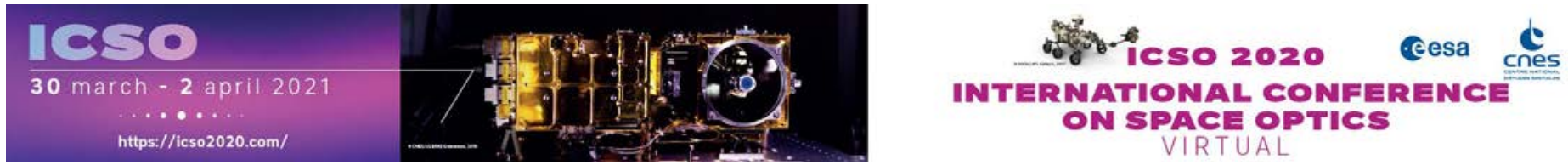

Characterization and video chain development of the CMOS
detector applied in the multi-angle spectro-polarimeter SPEXone

\section{- esa issporeatings denes}




\title{
Characterization and video chain development of the CMOS detector applied in the multi-angle spectro-polarimeter SPEXone
}

\author{
Jochen Campo*a, Paul Tol ${ }^{\mathrm{a}}$, Joris van der Vlugt ${ }^{\mathrm{a}}$, Martijn Smit ${ }^{\mathrm{a}}$, Jelle Talsma ${ }^{\mathrm{a}}$, \\ Jens Johansen ${ }^{\mathrm{a}}$, Alexander Eigenraam ${ }^{\mathrm{a}}$, Richard van Hees ${ }^{\mathrm{a}}$, Otto Hasekamp ${ }^{\mathrm{a}}$, Marc Oort ${ }^{\mathrm{b}}$, \\ Aaldert van Amerongen ${ }^{\mathrm{a}}$, and Jeroen Rietjens ${ }^{\mathrm{a}}$

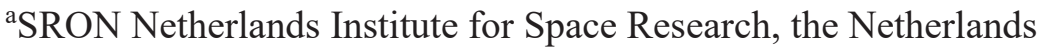 \\ ${ }^{\mathrm{b}}$ Airbus Defence and Space Netherlands (ADSN)
}

\begin{abstract}
This contribution presents the on-ground characterization and video chain development of the CMOS detector implemented in SPEXone, the five-angle space spectro-polarimeter for the NASA PACE observatory scheduled for launch in 2023. SPEXone is a Dutch compact payload contribution developed in a partnership between SRON and ADSN, and supported by TNO. Making use of spectral modulation, this polarimeter will enable in-depth and global characterization of the microphysical properties of fine particulate matter or aerosols in the atmosphere from low Earth orbit. In SPEXone, the spectrally modulated images are captured by means of a commercial-off-the-shelf detector module (DEM) from 3Dplus, which is equipped with a CMOS image sensor with integrated front-end-electronics. Video chain developments, including DEM firmware, read-out, flexible binning and DEM interfacing through SpaceWire have been carried out in-house. Making use of the firmware, the optimal detector parameters with associated random noise, full-well capacity, and photo response non-uniformity (PRNU) of the DEM were determined by placing the DEM in front of an integrating sphere fiber-fed with a stable white light source with accurately adjustable intensity and a highly linear reference detector, providing highly uniform illumination of the whole detector area at well-known relative light intensities. The rationale behind the measurement sequences is explained, and the full-well and read noise performance under different gain settings is described. The full-well capacity of the DEM is found to be not constant, but increasing significantly with illumination intensity.
\end{abstract}

Keywords: Polarimetry, aerosols, CMOS detector, detector video chain, pixel binning, detector characterization

\section{INTRODUCTION}

SPEXone ${ }^{1-4}$ is a five-angle space spectro-polarimeter developed in a partnership between SRON and ADSN, and supported by TNO, as a Dutch compact payload contribution to the NASA PACE observatory. Flying in low Earth orbit, SPEXone will enable in-depth, global characterization of the microphysical properties of fine particulate matter or aerosols in the atmosphere..$^{5-10}$ This is made possible by making use of spectral modulation ${ }^{11}$ to encode the linear polarization state (both degree and angle) of scattered sunlight directly in the continuous wavelength spectrum, and by combining the radiance and polarization information from the five distinct viewing angles. In SPEXone (instrument overview in Figure 1), the scattered sunlight is collected from the different angles by a stack of five compact three-mirror telescopes, mapping five push-broom swaths into a single spectrometer entrance slit, separated by masked out areas. Common polarization modulation optics $(\mathrm{PMO})^{1-3}$ are used to achieve spectral modulation in two pairs of five angular channels, which are next spectrally resolved by means of one and the same spectrometer.

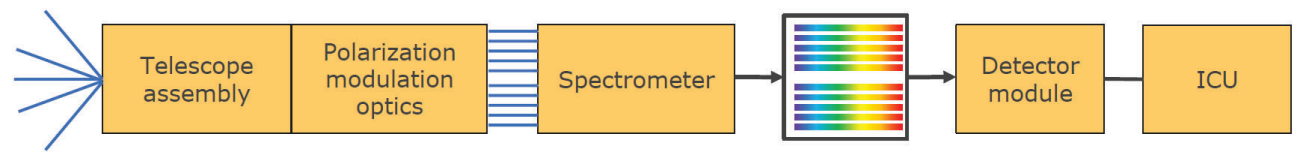

Figure 1. Instrument overview of SPEXone

The resulting ten spectral images are captured by means of a commercial-off-the-shelf 3D detector module (DEM) from 3Dplus in France, which is equipped with a 2048 x 2048 AMS CMV4000 CMOS image sensor with integrated front-end electronics containing a Microsemi FPGA and SDRAM and FLASH memories. The DEM was selected out of a large

J.Campo@sron.nl; phone+31(0)88 7775719 
batch in coordination with the vendor 3Dplus and the involved partners at CNES, mainly based on the provided values for random noise, full-well capacity, and dark current. In this contribution, the on-ground characterization (in unbinned / full frame mode) and video chain development of this DEM is presented.

The DEM was characterized stand-alone (early on in the project, prior to integration into the SPEXone instrument), as in this way uniform illumination of the whole detector area is possible with each pixel receiving the same amount of light, under the same conditions and with the same spectral content. This enables high-quality characterization measurements not possible with the telescope and spectrometer in front, and enables good interpretation of the results. In second instance, delta characterization of the DEM in the integrated instrument is also valuable, and needed (and is possible to a certain extent, using both external and internal light sources), but is not described here. Two DEMs were characterized sequentially, and the DEM with best performance was selected as the flight model DEM. The other DEM (also performing within requirements) was designated as the flight spare DEM. The results for the flight model DEM are presented here.

In brief, the optimal detector parameters and associated full-well capacity, random noise and photo response nonuniformity (PRNU) of the DEM were determined by means of an integrating sphere featuring a stable white light source with accurately adjustable intensity and a highly linear reference detector. The measurements were performed by making use of dedicated in-house video chain developments, including DEM firmware, read-out, flexible binning and DEM interfacing through SpaceWire. The measurement procedure followed to determine the optimal detector parameters is explained, and the achieved characterization results are presented. In particular, the observed dependence of the full-well capacity with illumination level, and the full-well and read noise performance under different gain settings is described.

\section{VIDEO CHAIN DEVELOPMENT}

\subsection{Detector Module (DEM)}

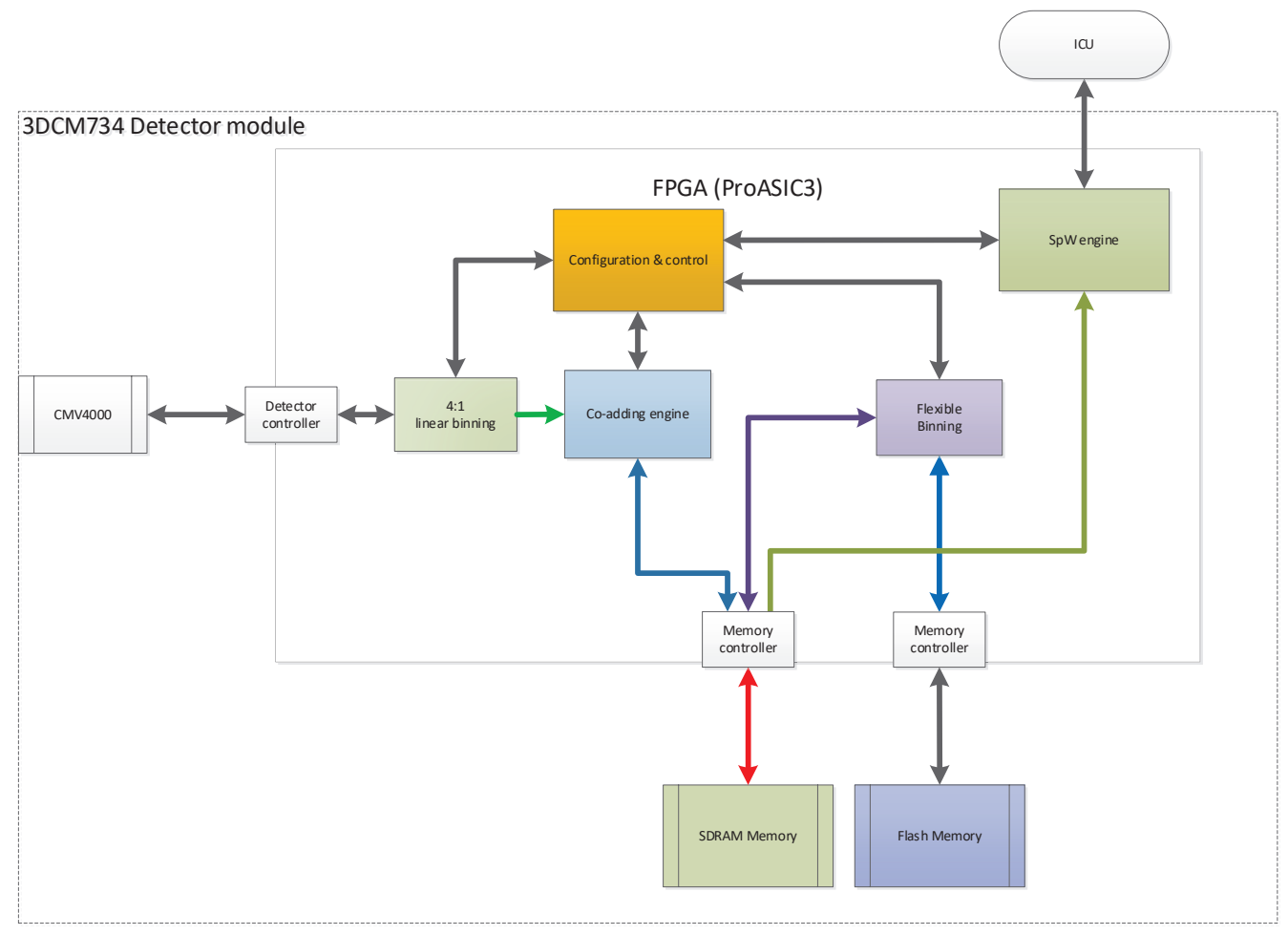

Figure 2. Overview of the video chain processing steps. Pixel data is received by the detector controller and binned to $2 \times 2$ super pixels. Co-adding is performed on a predefined set of consecutive images, while making extensive use of the SDRAM with multiple read-modify-write operations. The next processing step is the flexible binning. This allows for full control over which pixels are put into which bin. After completing the flexible binning, the fully processed image can be read out over the SpaceWire interface. 
The entire video chain (see Figure 2) resides in a single module: the DEM. This is a commercial-off-the-shelf module, the 3DCM734 by 3Dplus. It features a AMS CMV4000 image sensor with build-in front-end electronics, a Microsemi ProASIC3 FGPA, FLASH and SDRAM memories and power regulators in one single unit. The FPGA offers flexible use of the image sensor by allowing control over clocking, configuration and readout scheme. The SDRAM memory is used for intermediate storage while processing data, the FLASH memory is used for storage of flexible binning tables and line enable vectors. A single 5V power supply is used to power the module. Readout of the DEM is performed over a single SpaceWire link which can either interface with a STAR-Dundee SpaceWire Brick Mk3 for lab use and during development, or interface with the instrument's Instrument Control Unit (ICU). SRON developed the firmware for all FPGA data processing steps. For the SpaceWire interface the OpenCores SpaceWire Light core was used. Main limitation during development was the limited bandwidth available to the SDRAM: $800 \mathrm{Mbit} / \mathrm{s}$, in combination with the required 15 frames per second during observation in flight. The firmware is designed and tested according to tailored ECSS norms for spaceflight. With this firmware, the capability of the DEM is tailored to the SPEXone application making maximal use of resources.

\subsection{Data processing}

To make best use of the available resources several processing steps were implemented. The global design of the video chain is given in Figure 2. The detector is connected to the FPGA with 8 LVDS channels for pixel data. Collection of this data, combined with controlling the detector's registers and clock is taken care of by the detector controller. In the nominal observation mode the detector is triggered at 15 frames per second. The first processing step is to bin $2 \times 2$ pixels into one single super pixel. This is done to reduce the effective number of pixels to be processed from 4 megapixels to 1 megapixel, while combining $2 \times 2$ pixels still makes us of all pixel data for increased signal to noise performance. Combined with this 4:1 binning, also non-volatile stored, configurable line enable vectors are used to make sure only useful detector rows are processed. This further reduces the number of processed pixels. 5 consecutive $4: 1$ binned frames are co-added in the next processing step, with read-modify-write operation to the SDRAM. Reading the data from previous frames and co-adding the new frame on the fly is by far the most bandwidth intensive operation, using up to a maximum of $500 \mathrm{Mbit} / \mathrm{s}$ of the available $800 \mathrm{Mbit} / \mathrm{s}$.

To allow for maximum flexibility in spectral and spatial binning factors, and to be able to perform smile correction and bad-pixel removal, a fully configurable flexible binning step completes the video chain processing path. This flexible binning engine starts processing a co-added image after co-addition is completed. The timeframe in which it needs to finish is approximately the time it takes to complete the next co-addition, to make sure the entire processing chain does not run out of time. To determine which coadded pixel needs to be put in which bin, a binning table is used. This binning table resides in the non-volatile FLASH memory inside the DEM (see Figure 2). Such a binning table consists of a binning pointer for each individual pixel. A total of $1024^{2}$ of these pointers complete one binning table. Multiple binning tables can be stored simultaneously in the FLASH memory. These binning tables can be selected as part of the power-up configuration of the DEM. Uploading binning tables is thus not necessary after each power cycle. To ensure proper protection of the stored binning tables, a Hamming $(31,26)$ encoding algorithm is used. This algorithm allows for single bit error correction and double bit error detection for each binning pointer. To reduce the strain on the SDRAM interface the flexible binning engine incorporates a cache memory, since the SDRAM bandwidth needs to be shared with the coadder and the SpaceWire interface for readout. Complexity of the binning table will impact flexible binning performance: the more spread out the binning pointers are over the entire binned image, the more likely a cache miss is, which would result in the need for additional data transfers from the SDRAM. This limits the maximum complexity of the binning table in combination with a given detector frame rate. After completion, the binned image can be read out over a SpaceWire core running at $25 \mathrm{Mbit} / \mathrm{s}$. This readout needs to be finished before being overwritten by the image after next, due to a ping-pong buffer being used to store the result.

\subsection{Full frame readout}

Individual data of all 4 megapixels are required during the detector characterization, most of the on-ground instrument calibration and for monitoring in flight. Therefore the binning steps can be bypassed to allow for full frame readout. To support this the readout of the detector needs to be slowed down, to avoid overflowing the memory controller. This is done by using only 2 output channels of the detector instead of 8. 4:1 binning and flexible binning are disabled in full frame mode, but the co-adding feature is still available since it is a useful feature for various calibration and characterization measurements. 


\section{DETECTOR CHARACTERIZATION}

\subsection{Detector characterization setup}

The detector characterization was performed by making use of an integrating sphere equipped with a highly linear reference detector and fiber-fed by a stable white light source with accurately adjustable intensity. Placing the detector right in front of the sphere output port yields highly uniform illumination of the whole detector area at well-known relative light intensities, as needed for (non)linearity, random noise, and photo response non-uniformity (PRNU) measurements. The setup is described in detail below and is depicted in Figures 3 and 4.
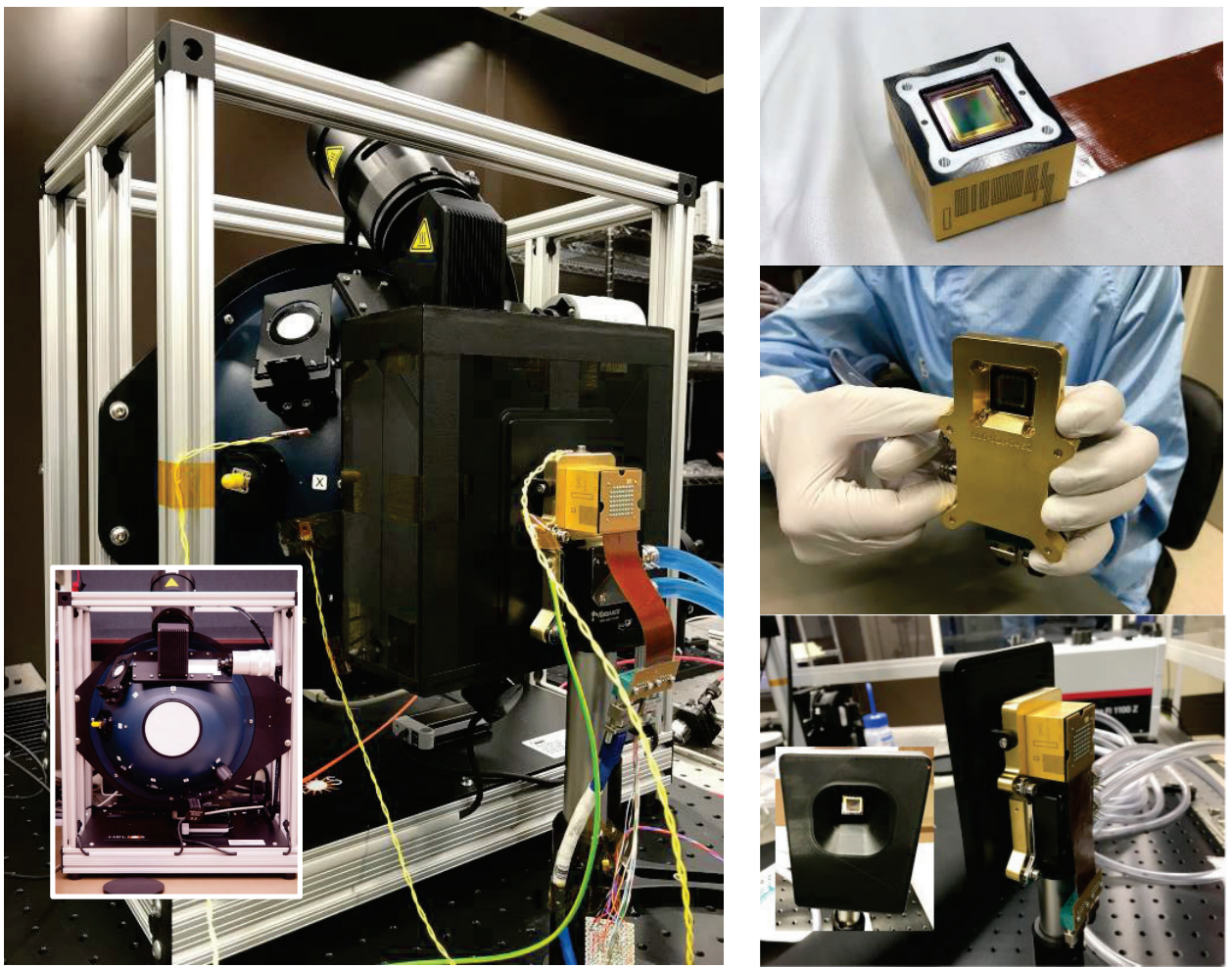

Figure 3. Pictures of the DEM and the characterization setup. Left: DEM mounted in front of the integrating sphere, also showing the DEM interfacing via SpaceWire (blue cable), the light-tight enclosure (black box) and the temperature sensors (yellow wires). The inset shows a head-on view of the output port of the sphere. Right, from top to bottom: the DEM itself, the DEM mounted from the front on the copper block for cooling, and the DEM with copper block, cooling block and black 3D printed part to connect to the enclosure. The inset shows how the 3D printed part connects to the front of the DEM.

As a stable white-light source, use was made of the fiber-coupled EQ-99X-FC-S Energetiq Laser-Driven Light Source (LDLS) from Hamamatsu. This electrodeless source provides highly intense, broadband light covering the spectral region of interest for SPEXone and beyond. The LDLS was coupled to a 12 inch diameter Spectraflect coated integrating sphere from Labsphere (CSTM-USLR-V12F), but not directly. As the light level of the LDLS is fixed, a dedicated attenuation setup was built to enable gradual variation of the light intensity as well as spectral pre-filtering in free space, prior to entering the sphere (see Figure 4, right panel). In brief, a reflective fiber collimator from Thorlabs (RC04FCP01) was used to collimate the light emerging from the LDLS output fiber. The collimated beam was then sent through (i) a Thorlabs FESH0750 premium cut-off filter blocking infrared light beyond $750 \mathrm{~nm}$ as well as ultraviolet light below $380 \mathrm{~nm}$, (ii) an electronic shutter (Uniblitz VS25) to facilitate background measurements, and (iii) an adjustable mechanical slit (Thorlabs VA100/M) enabling gradual attenuation of the transmitted light intensity. Finally, another reflective fiber collimator was used to re-couple the light into another fiber, going to a fiber port adapter at one of the side ports of the integrating sphere. The sphere has a built-in highly stable silicon photodiode sensor (Labsphere Helios SD-S1) with large linear dynamic range, which was used for monitoring variations in the overall (spectrally unresolved) light intensity, and to determine the light level step sizes taken during the measurements. 
For the characterization, the DEM was mounted directly in front of the output port of the integrating sphere, in an ISO 6 cleanroom. To shield the DEM from ambient light, a light-tight enclosure was used, closely connected to the edge of the output port and the front of the DEM. Whereas the integrating sphere has originally a 4 inch output port, for the DEM characterization this port was reduced to a 2 inch port using a port frame reducer (PFR-FM/M-400-200-SF), to somewhat restrict the solid angle at which the detector is exposed to light, thus increasing representativity with respect to the situation in the integrated instrument. To enable cooling, the DEM was mounted on a copper block, to which in turn a commercial cooling block (Koolance GPU-230) was connected which was cooled using a water chiller (NESLAB-RTE-7 circulating bath, Thermo-Scientific). Throughout the characterization, the chiller was set at $17^{\circ} \mathrm{C}$, in order to keep the DEM roughly at room temperature. Several temperature sensors (Fluke PT1000) were used to monitor the temperatures in the setup.
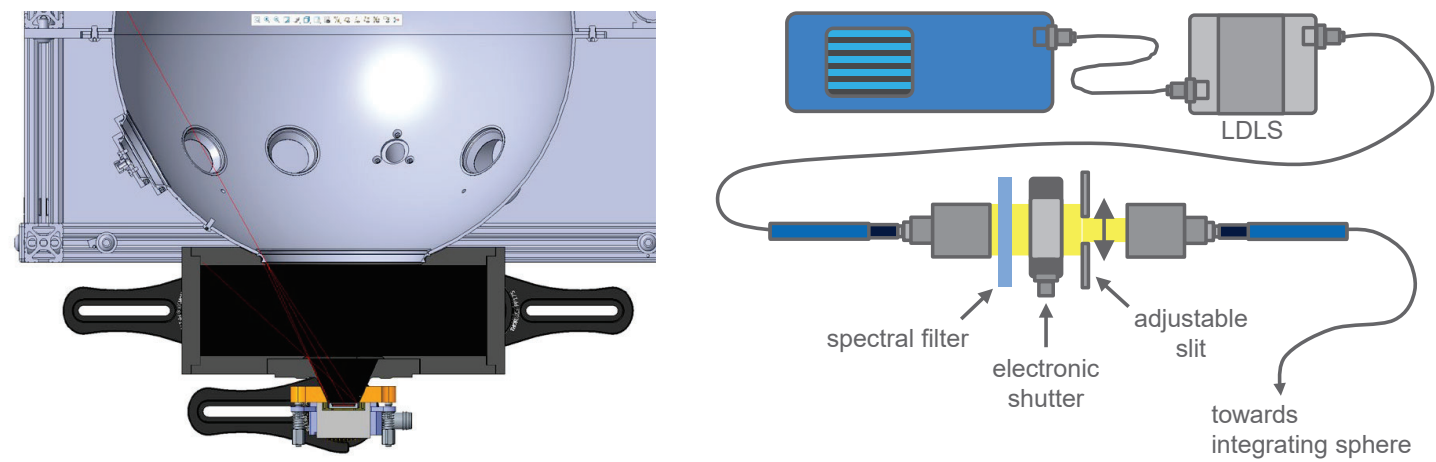

Figure 4. Left: CAD drawing (top view) of the DEM characterization setup, showing (from bottom to top) the mounted DEM, the light-tight box shielding the DEM from ambient light, and part of the integrating sphere. Right: Schematic representation of the attenuation setup to adjust the light level and pre-filter the light originating from the Laser-Driven Light Source (LDLS) before entering the integrating sphere.

\subsection{Detector parameters and characterization procedure}

\section{Detector parameters}

The signals received from the detector for a given light intensity are governed by four detector parameters: (i) the analog gain $g_{\text {pga }}$ which is applied by the programmable gain amplifier (PGA) to the pixel voltage, (ii) the gain of the analog-todigital converter (ADC) $r_{\text {adc }}$ which converts the voltage to a 10 bit (ADC resolution) signal value, (iii) the ramp voltage $r_{\text {ramp }}$ used by the ADC, and (iv) the digital offset $\Delta S$ added to the resulting digital signal (before clipping the signal to the $\mathrm{ADC}$ range of $0-1023$ ). The ADC is performed by linearly increasing a reference voltage and counting the number of time steps it takes for this voltage to increase from a starting value (ramp voltage $r_{\text {ramp }}$ ) until it reaches a reset voltage and next the pixel voltage. The difference between the two step numbers is the digital signal. As such, the signal value associated with a given pixel voltage is governed by the voltage step size, which is related to the ADC gain parameter.

In this contribution, the black level $S_{\mathrm{b}}$ and white level $S_{\mathrm{w}}$ are respectively defined as the final digital signal in the limits of short and long exposure times, at a given light intensity. To determine the black and white levels $S_{\mathrm{b}}$ and $S_{\mathrm{w}}$, the digital offset $\Delta S$ should be set such that they are not confined to the ADC range. The actual gain and the optimal settings depend on the master-clock frequency, which is set at $10 \mathrm{MHz}$.

\section{Photon Transfer Curve (PTC)}

The photon transfer curve (PTC) method ${ }^{12}$ is a quick way to determine several detector parameters from a series of measurements as a function of exposure time at a fixed light level, and modeling the detector noise (standard deviation of signal in digital counts). Within the PTC approach, it is assumed that the detector noise consists of:

1. Read noise, independent of signal strength

2. Photon shot noise, which increases with the square root of the signal strength (in electrons) 
3. Fixed pattern noise, which increases linearly with the signal strength (in electrons), with the Photo Response Non-Uniformity (PRNU) as scaling factor.

As such, noise $\sigma$ in digital counts can be written in terms of mean signal $S$ in digital counts as:

$$
\sigma^{2}=\left(g \cdot \sigma_{\mathrm{r}}\right)^{2}+g \cdot S+(p \cdot S)^{2}
$$

with conversion gain $g$ in counts per electron, read noise $\sigma_{\mathrm{r}}$ in electrons and PRNU factor $p$. The effects of PRNU and imperfect uniform illumination on the signal can be compensated, at least in the noise determination, when the difference between two successive frames is used. This method produces a single noise value for all pixels.

\section{Measurement procedure}

The DEM characterization was performed in unbinned / full frame mode. The following general measurement procedure was followed:

- First, the appropriate ramp voltage $r_{\text {ramp }}$ was determined by taking dark measurements at a fixed, short exposure time with large digital offset $\Delta S$ (low signals never clipped by ADC) at many combinations of $r_{\text {ramp, }} g_{\text {pga }}$ and $r_{\mathrm{adc}}$, and investigating the column-to-column variation of the black level $S_{\mathrm{b}}$.

- Next, using the selected $r_{\text {ramp, }}$, the white level $S_{\mathrm{w}}$ was measured at a fixed, high light intensity with large, negative digital offset (high signals never clipped by ADC) at many combinations of $g_{\text {pga }}$ and $r_{\text {adc. The full-well }}$ capacity is given by $S_{\mathrm{w}}-S_{\mathrm{b}}$, taking into account the different $\Delta S$ used in the determination of $S_{\mathrm{w}}$ and $S_{\mathrm{b}}$.

- Photon transfer curves (PTC) were measured at the same, high light intensity, at many combinations of $g_{\text {pga }}$ and $r_{\text {adc }}$, to select the optimal values for these parameters.

- Noise data were measured by measuring many frames at many exposure times, using the chosen combination of $\Delta S, r_{\text {ramp }}, g_{\text {pga }}$ and $r_{\text {adc. }}$.

- At a high, fixed light intensity, the PRNU was measured by taking many frames at a representative exposure time using the chosen combination of $\Delta S, r_{\text {ramp }}, g_{\text {pga }}$ and $r_{\text {adc }}$.

Noise and PTC measurements are not coadded, while white-level, black-level and PRNU measurements are coadded (images summed over time).

\subsection{Determination of optimal detector parameters}

\section{Ramp voltage $r_{\text {ramp }}$}

Before determining the best values for $g_{\text {pga }}, r_{\text {adc }}$ and $\Delta S$, the dependence on ramp voltage parameter $r_{\text {ramp }}$ was examined by measuring dark signals as a function of $r_{\text {ramp }}$ for large $\Delta S$ (low signals never clipped by ADC) and different combinations of $g_{\text {pga }}$ and $r_{\text {adc }}$. Upon increasing $r_{\text {ramp }}$, more and more low pixel signals get clipped, whereas the higher signals become lower until they also get clipped. As the higher signals vary more strongly from column to column than the lower signals, the column-to-column variation will increase with increasing $r_{\text {ramp }}$, until the clipping starts to take over. An as high as possible $r_{\text {ramp }}$ value before deterioration takes place was selected (104).

\section{Black level, white level and illumination level dependent full-well capacity}

The detector black level $S_{\mathrm{b}}$ was determined with the light source off and short exposure time. The white level $S_{\mathrm{w}}$ was measured at a fixed, high light intensity. From this, the full-well capacity can be calculated (see Table 1), as this quantity is given by the difference between the final digital signals in the limits of short and long exposure times, i.e. $S_{\mathrm{w}}-S_{\mathrm{b}}$, at a given light intensity. Both the black and white levels increase at larger gain parameters $g_{\text {pga }}$ and $r_{\text {adc }}$, and they are also affected by $r_{\text {ramp. }}$.

Table 1. Detector median of the full-well capacity (right), in counts and as a function of gain parameters $g_{\text {pga }}$ and $r_{\text {adc. }}$.

\begin{tabular}{rrrrrrrrrrrrr} 
& & & & & & \multicolumn{7}{c}{$r_{\text {adc }}$} \\
\hline \multirow{4}{*}{$g_{\text {pga }}$} & 51 & 52 & 53 & 54 & 55 & 56 & 57 & 58 & 59 & 60 & 61 \\
\hline & 1.0 & - & - & - & - & - & - & 645 & 755 & 913 & clipped & clipped \\
& 1.2 & - & - & - & - & 619 & 698 & 799 & 936 & clipped & clipped & - \\
& 1.4 & - & - & - & 639 & 709 & 798 & 914 & clipped & clipped & - & - \\
& 1.6 & - & 575 & 625 & 684 & 759 & 856 & 972 & clipped & clipped & - & - \\
& 2.0 & 558 & 600 & 651 & 713 & 789 & 888 & 1008 & clipped & - & - & - \\
\hline
\end{tabular}


Interestingly and counter-intuitively, the full-well capacity of the DEM is not a constant, but increases logarithmically with light intensity. ${ }^{13,14}$ Two extreme cases for very different light intensities are shown in Figure 5.
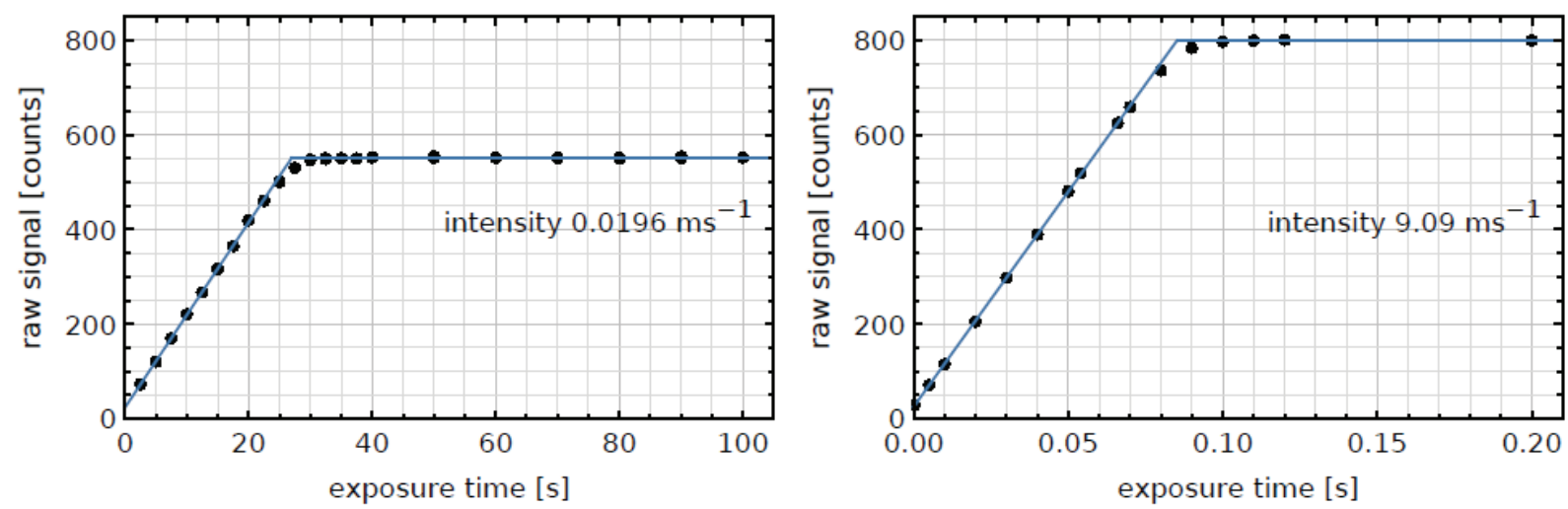

Figure 5. Detector signal as a function of exposure time at two disparate light intensities, showing the marked difference in full-well capacity.

This also means that, whereas the signal at a fixed intensity reaches a constant value at large exposure times, the signal at a fixed exposure time keeps increasing at large intensities (see Figure 6).

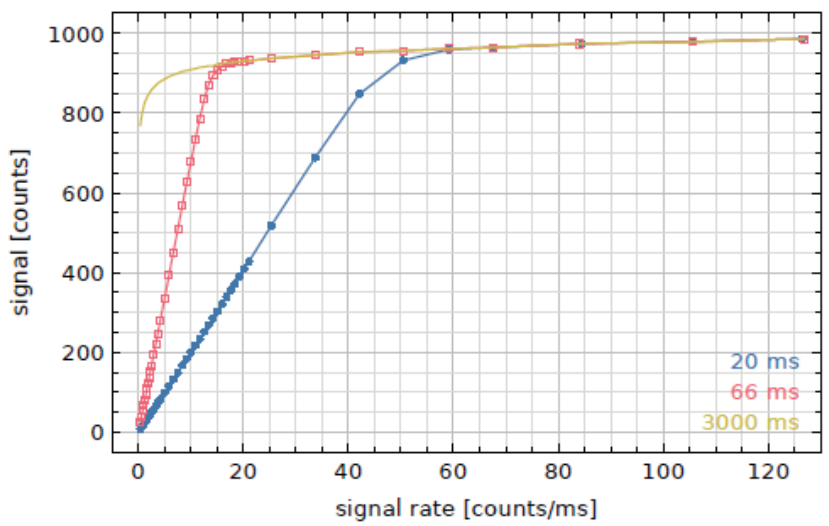

Figure 6. Detector median of the signal as a function of intensity (given as a signal rate) at several exposure times. The data at $3000 \mathrm{~ms}$ exposure time show the maximum possible signal depending on intensity. The used gain values are $\left(g_{\text {pga }}, r_{\text {adc }}\right)=(57,1.4)$.

\section{Analog and PGA gains $r_{\text {adc }}$ and $g_{\text {pga }}$}

To determine the best values for $g_{\text {pga }}$ and $r_{\text {adc }}$, Photon Transfer Curve (PTC) measurements (see above) were performed at many combinations of the gain parameters. The sensitivity and noise behavior was determined by taking frame pairs at many different exposure times, at the fixed light intensity also used for the white level determination. The frames within each pair were subtracted from each other to eliminate contributions from fixed pattern noise and imperfect uniform illumination, and the accordingly converted expression (1) was fitted to the data (see Figure 7). The obtained read noise and inverse conversion gains at the different combinations of $g_{\text {pga }}$ and $r_{\text {adc }}$ are listed in Table 2 . In a few cases, a full noise determination per detector pixel was performed using data at varying light levels. The median values obtained as such (not shown) are almost the same as the values from the PTC method.

In order to achieve the least noise and highest sensitivity (lowest conversion gain), the two gain parameters should be as high as possible. However, this does not take into account that at high gains the signal will be clipped by the ADC range. A graphical way to inspect the effect of the gain settings is to plot the signal range in electrons against the inverse conversion gain (see Figure 8). When the signal is not clipped by the ADC, the signal range is the full-well capacity. The cases where the signal is clipped form a diagonal line where the slope is the (detector median of the) maximum signal 
range expressed in counts, in this case on average 968. Apparently, the signal range increases with decreasing PGA gain and the inverse of the conversion gain decreases with increasing ADC gain. The reason for this behavior is not clear. Note that for all gain combinations, the measured signal range is significantly lower than the full well capacity of $13.5 \mathrm{ke}^{-}$specified for this sensor. Since the full well capacity increases with the illumination level, the observed difference could be related to a different test environment. However, the illumination level at which the $13.5 \mathrm{ke}^{-}$was measured according to the specification is not specified. Another difference in test environment is the operating frequency of $10 \mathrm{MHz}$, instead of the nominal $48 \mathrm{MHz}$ for this sensor. Operating at $10 \mathrm{MHz}$ requires the use of different gain settings than at $48 \mathrm{MHz}$, and as can be seen in Figure 8, the signal range depends strongly on the gain parameters.
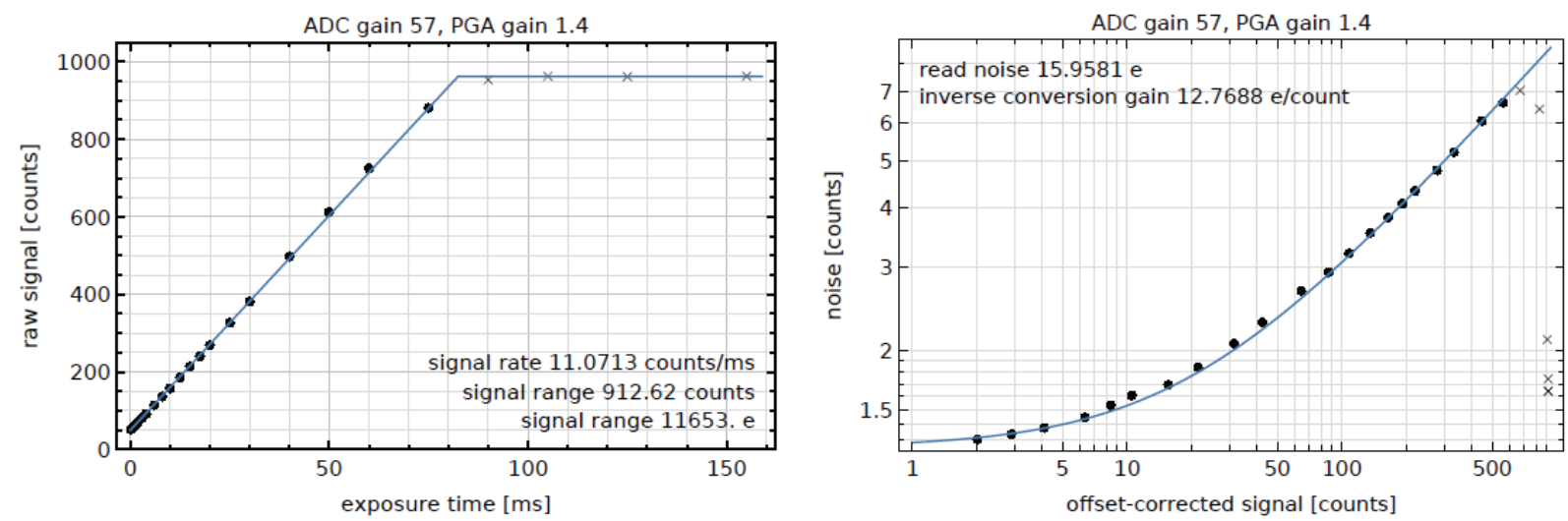

Figure 7. Example of a noise measurement, at the gain combination specified above the panels. Left: raw signal (not corrected for digital or fitted offset) as a function of exposure time. Right: noise as a function of signal corrected for the offset fitted in the left panel. Data included and excluded in the fits are shown as points and crosses, respectively. Linear fit functions are used in the left panel, expression (1) is used in the right panel. The fitted signal rate, signal range, read noise and inverse of the conversion gain are given within the panels.

Table 2. Detector median of the read noise [e] (left) and the inverse conversion gain [e/count] (right), as a function of gain parameters $g_{\text {pga }}$ and $r_{\text {adc. }}$.

\begin{tabular}{|c|c|c|c|c|c|c|c|c|c|c|c|c|}
\hline & \multicolumn{11}{|c|}{$r_{\mathrm{adc}}$} \\
\hline & & 51 & 52 & 53 & 54 & 55 & 56 & 57 & 58 & 59 & 60 & 61 \\
\hline \multirow{5}{*}{$g_{\text {pga }}$} & 1.0 & - & - & - & - & - & - & 17.6 & 17.9 & 17.3 & 16.6 & 15.0 \\
\hline & 1.2 & - & - & - & - & 17.9 & 17.4 & 16.7 & 16.2 & 15.8 & 15.2 & - \\
\hline & 1.4 & - & - & - & 16.9 & 17.2 & 16.0 & 16.0 & 15.6 & 14.7 & - & - \\
\hline & 1.6 & - & 17.3 & 17.3 & 16.8 & 15.6 & 15.9 & 15.3 & 14.8 & 14.0 & - & - \\
\hline & 2.0 & 16.7 & 16.0 & 16.1 & 15.5 & 15.9 & 15.5 & 15.2 & 14.2 & - & - & - \\
\hline
\end{tabular}

\begin{tabular}{|c|c|c|c|c|c|c|c|c|c|c|c|c|}
\hline & \multicolumn{11}{|c|}{ radc } \\
\hline & & 51 & 52 & 53 & 54 & 55 & 56 & 57 & 58 & 59 & 60 & 61 \\
\hline \multirow{5}{*}{$g_{\text {pga }}$} & 1.0 & - & - & - & - & - & - & 17.9 & 16.0 & 13.3 & 10.7 & 7.5 \\
\hline & 1.2 & - & - & - & - & 19.2 & 17.0 & 14.9 & 12.8 & 10.7 & 8.5 & - \\
\hline & 1.4 & - & - & - & 17.9 & 16.5 & 14.4 & 12.8 & 11.0 & 9.0 & - & - \\
\hline & 1.6 & - & 19.1 & 17.6 & 16.2 & 14.2 & 12.7 & 11.1 & 9.6 & 7.8 & - & - \\
\hline & 2.0 & 18.3 & 16.9 & 15.7 & 14.1 & 13.1 & 11.6 & 10.3 & 8.6 & - & - & - \\
\hline
\end{tabular}

The two best settings are $\left(g_{\text {pga }}, r_{\text {adc }}\right)=(1.2,58)$ and $(1.4,57)$, as they have the largest dynamic range (ratio of signal range and read noise) of 740 and 730 , respectively (see Table 3), and at the same time a high sensitivity (low inverse conversion gain). The median read noise itself is 16.5 and 15.9 electrons, respectively. As a lower noise is important at small signals, setting $(1.4,57)$ was chosen as most optimal. Note that the measured read noise is higher than the specification of $13 \mathrm{e}^{-}$for this sensor. Similar to the difference in full-well capacity, this could be related to differences in operating frequency and associated gain parameters.

Table 3. Detector median of the dynamic range (ratio of signal range and read noise), as a function of the gain parameters $g_{\text {pga }}$ and $r$ adc.

\begin{tabular}{|c|c|c|c|c|c|c|c|c|c|c|c|c|}
\hline & \multicolumn{11}{|c|}{$r_{\text {adc }}$} \\
\hline & & 51 & 52 & 53 & 54 & 55 & 56 & 57 & 58 & 59 & 60 & 61 \\
\hline \multirow{5}{*}{$g_{\text {pga }}$} & 1.0 & - & - & - & - & - & - & 654 & 675 & 704 & 621 & 480 \\
\hline & 1.2 & - & - & - & - & 664 & 679 & 710 & 740 & 654 & 540 & - \\
\hline & 1.4 & - & - & - & 677 & 684 & 719 & 730 & 685 & 593 & - & - \\
\hline & 1.6 & - & 633 & 636 & 660 & 689 & 687 & 706 & 623 & 541 & - & - \\
\hline & 2.0 & 614 & 634 & 635 & 651 & 652 & 668 & 658 & 589 & - & - & - \\
\hline
\end{tabular}




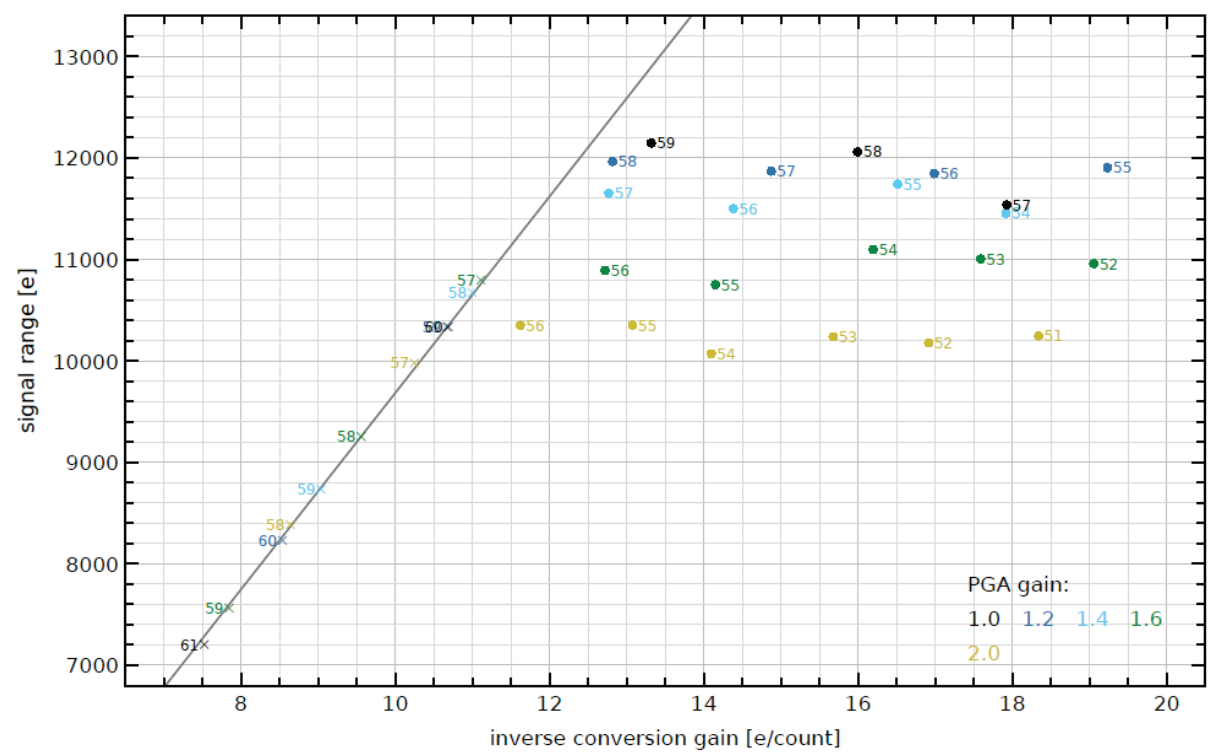

Figure 8. Detector median of the signal range as a function of the inverse of the conversion gain, both determined from fits to noise data at many pairs of ADC and PGA gain, at a fixed (high) light intensity. Unclipped signal ranges, shown as points, are values of the full-well capacity at the used light intensity. Signal ranges clipped by the ADC range, shown as crosses, form a diagonal line with a slope of 968 counts (less than 1023 due to offset and median values). The ADC gain parameter is given as a label near the symbols and the PGA gain is indicated by a color.

\subsection{Detector PRNU}

The Photo Response Non-Uniformity (PRNU) of the detector was determined using an offset-corrected measurement with a signal far below the maximum. The PRNU is given by the mean over the different frames divided by the detector median, under the premise that the integrating sphere illuminates the whole detector area uniformly. The resulting PRNU map is shown in Figure 9. The standard deviation is 0.011.

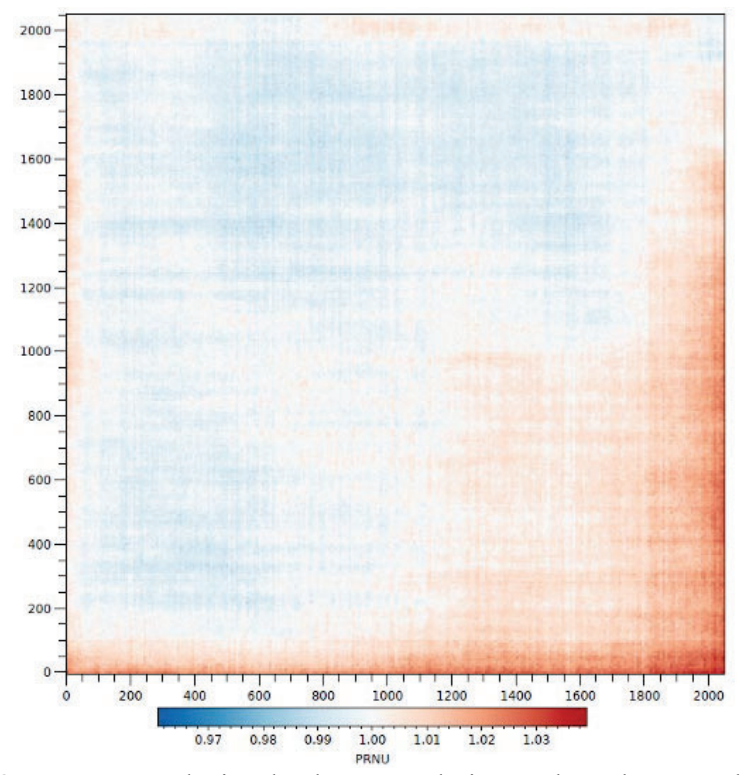

Figure 9. PRNU map, depicted at lower resolution to show the general pattern. 


\section{CONCLUSIONS}

The video chain of the SPEXone DEM has been developed, enabling its optical characterization by using an integrating sphere with stable white-light source and reference photodiode sensor. By strategically performing measurements at varying detector parameters and as a function of exposure time and/or light level, the optimal parameter values were determined (analog gain $g_{\text {pga }}=1.4$, ADC gain $r_{\text {adc }}=57$, ramp voltage $r_{\text {ramp }}=104$, digital offset $\Delta S=25$, at a master-clock frequency of $10 \mathrm{MHz}$ ). At these preferred settings, the detector performance was measured in terms of read noise $\left(15.9 \mathrm{e}^{-}\right)$, sensitivity (inverse conversion gain: $12.7 \mathrm{e}^{-} /$count), full-well capacity $(11.6 \mathrm{ke}$, but found to be logarithmically dependent on light intensity (see Figure 6)), dynamic range (730), and PRNU ( $\pm 1 \%$ standard deviation). The obtained full-well capacity is significantly larger and the measured read noise is lower than the initially budgeted values in SPEXone performance calculations, which were based on limited measurements on a breadboard version of the DEM. With this, the SPEXone DEM performs better than anticipated, which will benefit the performance of the integrated SPEXone instrument.

\section{ACKNOWLEDGMENTS}

SPEXone is a public-private initiative of SRON Netherlands Institute for Space Research and Airbus Defence and Space Netherlands, supported by opto-mechanical expertise from TNO. We acknowledge funding by the Ministry of Education, Culture and Science (OCW), the Netherlands Space Office (NSO), and the Netherlands Organization of Scientific Research (NWO). We acknowledge NASA for hosting SPEXone on their PACE observatory as a partnered payload. We also acknowledge 3Dplus, and Alain Bardoux and Cédric Virmontois at CNES for interactions and advice in selecting the DEM.

\section{REFERENCES}

[1] Rietjens, J., Campo, J., Chanumolu, A., Smit, M, Nalla, R., Fernandez, C., Dingjan J., van Amerongen, A., and Hasekamp, O., "Expected performance and error analysis for SPEXone, a multi-angle channeled spectropolarimeter for the NASA PACE mission", Proc. SPIE 11132 (2019), doi: 10.1117/12.2530729.

[2] van Amerongen, A., Rietjens, J., Campo, J., Dogan, E., Dingjan, J., Nalla. R., Caron, J., and Hasekamp, O., "SPEXone: a compact multi-angle polarimeter", proc. SPIE 111800L (2019), doi: 10.1117/12.2535940.

[3] Rietjens, J., Campo, J., Smit, M., Winkelman, R., Nalla, R., Landgraf, J., Hasekamp, O., Oort, M., van Amerongen, A., and SPEXone team, "Optical and system performance of SPEXone, a multi-angle channeled spectropolarimeter for the NASA PACE mission", in [International Conference on Space Optics - ICSO 2020], International Society for Optics and Photonics, SPIE (2021).

[4] Smit, M., Rietjens, J., Campo, J., aan de Brugh, J., van Amerongen, A, Hasekamp, O., Landgraf, J., and Liu, X., "Polarimetric calibration of a spectro-polarimeter for remote sensing and characterization of aerosols", in [International Conference on Space Optics - ICSO 2020], International Society for Optics and Photonics, SPIE (2021).

[5] Hansen, J.E. and Travis, D.E., "Light Scattering in Planetary Atmospheres", Space Science Reviews 16, 527 (1974).

[6] Mishchenko, M.I. and Travis, L.D., "Satellite retrieval of aerosol properties over the ocean using polarization as well as intensity of reflected sunlight", Journal of Geophysical Research - Atmospheres, 102(D14), 16989 (1997).

[7] Kokhanovsky, A.A., Deuze, J.L., Diner, D.J., et al., "The inter-comparison of major satellite aerosol retrieval algorithms using simulated intensity and polarization characteristics of reflected light", Atmospheric Measurement Techniques, 3(4), 1005 (2010).

[8] Hasekamp, O.P. and Landgraf, J., "Retrieval of aerosol properties over land surfaces: capabilities of multipleviewing-angle intensity and polarization measurements", Applied Optics, 46(16), 3332 (2007).

[9] Wu, L., Hasekamp, O.P., Diedenhoven, B.v., and Cairns, B., "Aerosol retrieval from multiangle, multispectral photopolarimetric measurements: Importance of spectral range and angular resolution", Atmospheric Measurement Techniques 8(6), 2625 (2015). 
[10]Xu, F., van Harten, G., Diner, D.J., Kalashnikova, O.V., Seidel, F.C., Bruegge, C.J., and Dubovik, O., "Coupled retrieval of aerosol properties and land surface reflection using the Airborne Multi-angle Spectro-Polarimetric Imager", Journal of Geophysical Research - Atmospheres, 122(13), 7004 (2017).

[11] Snik, F., Karalidi, T., and Keller, C.U., "Spectral modulation for full linear polarimetry", Applied Optics, 48(7), 1337 (2009).

[12] Janesick, J. R., [Photon Transfer], Chapter 5: Photon Transfer Curve, SPIE digital library (2007), doi: 10.1117/3.725073.ch5.

[13] Pelamatti, A., Goiffon, V., Estribeau, M., Cervantes, P., and Magnan, P., "Estimation and Modeling of the Full Well Capacity in Pinned Photodiode CMOS Image Sensors", IEEE Electron Device Letters, 34(7), 900-902 (2013), doi: 10.1109/LED.2013.2260523.

[14] Pelamatti, A., Belloir, J.-M., Messien, C., Goiffon, V., Estribeau, M., Magnan, P., Virmontois, C., Saint-Pé, O., and Paillet, P., "Temperature Dependence and Dynamic Behavior of Full Well Capacity in Pinned Photodiode CMOS Image Sensors", IEEE Transactions on Electron Devices, 62(4), 1200-1207 (2015), doi: 10.1109/TED.2015.2400136. 